\title{
LATTICE PACKING OF NEARLY-EUCLIDEAN BALLS IN SPACES OF EVEN DIMENSION
}

\author{
by J. A. RUSH* \\ (Received 29th August 1994)
}

We consider nearly-Euclidean balls of the shape

$$
\left(x_{1}^{2}+x_{2}^{2}\right)^{1+\varepsilon}+\left(x_{3}^{2}+x_{4}^{2}\right)^{1+\varepsilon}+\cdots+\left(x_{n-1}^{2}+x_{n}^{2}\right)^{1+\varepsilon}<1
$$

where $\varepsilon$ is a small positive number, and $n$ is even. If $\varepsilon$ is small enough, then the maximum lattice-packing density of this body is essentially greater than the Minkowski-Hlawka bound for large $n$.

1991 Mathematics subject classification.11H31: Number theory; Geometry of Numbers; Lattice Packing

\section{Introduction}

Let $G$ be a convex $O$-symmetric body in $\mathbf{R}^{n}$, and $\delta_{L}(G)$ its maximum lattice-packing density. The Minkowski-Hlawka bound [7] assures us that

$$
\delta_{L}(G) \geq 2^{1-n \zeta(n)}
$$

for each integer $n$ greater than or equal to two. Thus, for large $n$,

$$
\delta_{L}(G) \geq 2^{-n(1+o(1))}
$$

For specific bodies it is sometimes possible to replace the exponential $2^{-n}$ with an exponential function of $n$ which decays less rapidly. For example, for the body

we have

$$
x_{1}^{4}+x_{2}^{4}+\cdots+x_{n}^{4}<1
$$

$$
\delta_{L} \geq 2^{-0.67424266 \ldots n(1+o(1))} \text {, }
$$

as was found in [11]. Since this multiplication of the Minkowski-Hlawka density by an exponential factor is more important than the discarded factor $2 \zeta(n)$ which was

*Supported by National Science Foundation grant DMS-9103233. 
absorbed by the error term, it is usual to call it an essential improvement. Improvements on the lattice-packing density of a given body by a factor which grows more slowly than any exponential are called nonessential.

In [4], an essential improvement to the Minkowski-Hlawka density was obtained for the ball

$$
\left|x_{1}\right|^{\sigma}+\left|x_{2}\right|^{\sigma}+\cdots+\left|x_{n}\right|^{\sigma}<1
$$

for each real $\sigma$ greater than 2. The present paper follows the procedure used there for the case $\sigma=2+\varepsilon$, insofar as this is permitted by the new complications which arise.

We shall prove this result:

Theorem. If the positive number $\varepsilon$ is sufficiently small, then the nearly-Euclidean ball defined in the abstract admits an essential improvement to the Minkowski-Hlawka bound.

It seems possible that the theorem remains true not only for small $\varepsilon$, but for all positive epsilon, but our proof does not show this. (See the table.) Moreover, it is probably possible, with more effort, to treat quite general nearly-Euclidean balls of the shape

$$
\left(x_{1}^{2}+\cdots+x_{k}^{2}\right)^{1+\varepsilon}+\left(x_{k+1}^{2}+\cdots+x_{2 k}^{2}\right)^{1+\varepsilon}+\cdots+\left(x_{n-k+1}^{2}+\cdots+x_{n}^{2}\right)^{1+\varepsilon}<1,
$$

where $k$ divides $n$, and volume given by

$$
\frac{\pi^{n / 2}}{\Gamma\left(1+\frac{n}{2} \frac{1}{1+\varepsilon}\right)}\left\{\frac{\Gamma\left(1+\frac{k}{2} \frac{1}{1+\varepsilon}\right)}{\Gamma\left(1+\frac{k}{2}\right)}\right\}^{n / k}
$$

but here we make no attempt to do so.

\section{Preliminaries}

Let

and

so that

$$
g(x)=g\left(x_{1}, x_{2}\right)=\left(x_{1}^{2}+x_{2}^{2}\right)^{1+\varepsilon}
$$

$$
f(x)=f\left(x_{1}, \ldots, x_{n}\right)=g\left(x_{1}, x_{2}\right)+g\left(x_{3}, x_{4}\right)+\cdots+g\left(x_{n-1}, x_{n}\right)
$$

$$
G=\left\{x \in \mathbf{R}^{n}: f(x)<1\right\}
$$

is the body under consideration. The volume of $G$ is 


\begin{tabular}{cll}
$\varepsilon$ & $t$ & $\sqrt{\frac{\int_{x_{1}=-\infty}^{\infty} \int_{x_{2}=-\infty}^{\infty} \exp \left(-\left(t^{2} x_{1}^{2}+t^{2} x_{2}^{2}\right)^{1+\varepsilon}\right) d x_{2} d x_{1}}{\sum_{x_{1}=-\infty}^{\infty} \sum_{x_{2}=-\infty}^{\infty} \exp \left(-\left(t^{2} x_{1}^{2}+t^{2} x_{2}^{2}\right)^{1+\varepsilon}\right)}}$ \\
\hline $1 / 1000$ & 0.8717866971783 & 1.0000199223957 \\
$1 / 100$ & 1.012069610695 & 1.0003797729768 \\
$1 / 10$ & 1.235107979915 & 1.009465385326 \\
1 & 1.31539855203 & 1.15778133146 \\
2 & 1.24356276816 & 1.28474664325 \\
3 & 1.19389418169 & 1.3699454725 \\
9 & 1.08743468370 & 1.5748604946 \\
99 & 1.01050144672 & 1.7479678259
\end{tabular}

Table. For each given $\varepsilon$, the positive number $t$ provides a global maximum for the elaborate expression at the head of the third column. That maximum exceeds unity in each case, providing an essential improvement to the Minkowski-Hlawka bound for the ball defined in the abstract. Decimal numbers are shown truncated, not rounded.

$$
\operatorname{vol}(G)=\frac{\pi^{n / 2} \Gamma\left(1+\frac{1}{1+\varepsilon}\right)^{n / 2}}{\Gamma\left(1+\frac{n}{2} \frac{1}{1+\varepsilon}\right)} .
$$

Equation (1) can be established by various means. The easiest seems to be to evaluate the integral over $\mathbf{R}^{n}$ of $\exp (-f(x))$ in two different ways and equate the results, as in [4] and [13]. The methods used in those articles also give, in the present case,

$$
\delta_{L}(G) \geq\left(\frac{1}{2} \sup _{A} \sqrt{\frac{\int \exp (-g(A x)) d V}{\sum \exp (-g(A x))}}\right)^{n(1+o(1))}
$$

in which $x=\left(x_{1}, x_{2}\right), d V=d x_{1} d x_{2}$, the integral is over all $x$ in $\mathbf{R}^{2}$, the sum is over all $x$ in the lattice $\mathbf{Z}^{2}$ of points with integer coordinates, and the supremum is taken over all nonsingular linear transformations $A: \mathbf{R}^{2} \rightarrow \mathbf{R}^{2}$. (In [13] the author conjectured that (2) holds with equality for a large class of functions $g$.)

Inequality (2) is our point of departure.

\section{Proof of theorem}

The plan is to estimate the ratio of sum to integral in (2), when $A$ is the dilation

$$
\left(x_{1}, x_{2}\right) \rightarrow\left(t x_{1}, t x_{2}\right)
$$

and $t>0$ is a function of $\varepsilon$ to be specified to our advantage later. The ratio will exceed 1 , giving the claimed essential improvement.

Under the square-root sign in (2) we have

$$
\frac{\int_{x_{1}=-\infty}^{\infty} \int_{x_{2}=-\infty}^{\infty} \exp \left(-\left(t^{2} x_{1}^{2}+t^{2} x_{2}^{2}\right)^{1+\varepsilon}\right) d x_{2} d x_{1}}{\sum_{x_{1}=-\infty}^{\infty} \sum_{x_{2}=-\infty}^{\infty} \exp \left(-\left(t^{2} x_{1}^{2}+t^{2} x_{2}^{2}\right)^{1+t}\right)}
$$


or

$$
\frac{\int_{x_{1}=-\infty}^{\infty} \int_{x_{2}=-\infty}^{\infty} \exp \left(-\left(x_{1}^{2}+x_{2}^{2}\right)^{1+\varepsilon}\right) d x_{2} d x_{1}}{t^{2} \sum_{x_{1}=-\infty}^{\infty} \sum_{x_{2}=-\infty}^{\infty} \exp \left(-\left(t^{2} x_{1}^{2}+t^{2} x_{2}^{2}\right)^{1+\varepsilon}\right)}
$$

In (3), denote the numerator by $\int$ and the denominator by $\sum$. As $t$ approaches zero, $\int / \sum \rightarrow 1$, because $\sum$ is a Riemann sum for $\int$. Note, incidentally, that $\int$ can be evaluated explicitly:

$$
\begin{gathered}
\int=\int_{\left(x_{1}, x_{2}\right) \in \mathrm{R}^{2}}\left(\int_{z=\left(x_{1}^{2}+x_{2}\right)^{1+\varepsilon}}^{\infty+\varepsilon} e^{-z} d z\right) d x_{2} d x_{1} \\
=\int_{z=0}^{\infty} e^{-z}\left(\int_{\substack{\left(x_{1}, x_{2}\right) \in \mathbf{R}^{2} \\
\left(x_{1}^{+}+x_{2}\right)^{1+\varepsilon<z}}} d x_{2} d x_{1}\right) d z \\
=\int_{z=0}^{\infty} e^{-z^{1 /(1+\varepsilon)} \operatorname{Vol}\left(x_{1}^{2}+x_{2}^{2}<1\right) d z} \\
=\pi \Gamma\left(1+\frac{1}{1+\varepsilon}\right) .
\end{gathered}
$$

write

$$
\frac{\int}{\sum}=1-\frac{\sum-\int}{\sum}
$$

Since $\sum$ is positive, we will be finished if $\sum-\int$ can be made negative.

By Poisson summation,

$$
\sum-\int=\sum_{\substack{k_{1}, k_{2}=-\infty \\\left(k_{1}, k_{2}\right) \neq(0,0)}}^{\infty} \Phi\left(k_{1} / t, k_{2} / t\right)
$$

where $\Phi$ is the Fourier transform of $\exp \left(-\left(v_{1}^{2}+v_{2}^{2}\right)^{1+\varepsilon}\right)$, that is

$$
\begin{aligned}
\Phi\left(w_{1}, w_{2}\right) & =\int_{v_{1}=-\infty}^{\infty} \int_{v_{2}=-\infty}^{\infty} e^{-\left(v_{1}^{2}+v_{2}^{2}\right)^{1+\varepsilon}} e^{2 \pi i\left(w_{1} v_{1}+w_{2} v_{2}\right)} d v_{2} d v_{1} \\
& =\int_{v \in \mathbf{R}^{2}} \exp \left(-\left(v_{1}^{2}+v_{2}^{2}\right)^{1+\varepsilon}\right) \cos (2 \pi v \cdot w) d V
\end{aligned}
$$

in which $v=\left(v_{1}, v_{2}\right), w=\left(w_{1}, w_{2}\right)$, and $d V=d v_{1} d v_{2}$. Letting $b=v_{1}^{2}+v_{2}^{2}$ and expanding $\exp \left(-b^{1+\varepsilon}\right)$ in powers of $\varepsilon$, we get

$$
e^{-b}-\varepsilon \frac{b \log b}{e^{b}}+\varepsilon^{2} \frac{(-1+b) b(\log b)^{2}}{2 e^{b}}+\varepsilon^{3} \frac{\left(-1+3 b-b^{2}\right) b(\log b)^{3}}{6 e^{b}}+\cdots
$$




$$
=e^{-b}-\varepsilon \frac{b \log b}{e^{b}}+R
$$

say. Fix any real number $c>1$, and integer $m>1$. Then $\varepsilon^{-2} R$ and its first $m$ partials are uniformly $O\left(\exp \left(-\left(v_{1}^{2}+v_{2}^{2}\right) / c\right)\right)$ for $b$ bounded strictly away from the origin and $\varepsilon$ bounded strictly away from infinity, so the Fourier transform of $\varepsilon^{-2} R$ is $O\left(\left(w_{1}^{2}+w_{2}^{2}\right)^{-3 / 2}\right)$. The Fourier transform of $e^{-b}$ is

$$
\int_{v \in \mathbb{R}^{2}} e^{-\left(v_{1}^{2}+v_{2}^{2}\right)} e^{2 \pi i v \cdot w} d V=\pi \exp \left(-\left(\pi^{2} w_{1}^{2}+\pi^{2} w_{2}^{2}\right)\right)
$$

and the Fourier transform of $(b \log b) / e^{b}$ is

$$
\xi=\int_{v_{1}}^{\infty} \int_{-\infty}^{\infty} e_{v_{2}=-\infty}^{-\left(v_{1}^{2}+v_{2}^{2}\right)}\left(v_{1}^{2}+v_{2}^{2}\right) \log \left(v_{1}^{2}+v_{2}^{2}\right) \cos \left(2 \pi\left(v_{1} w_{1}+v_{2} w_{2}\right)\right) d v_{2} d v_{1}
$$

as a function of the rectangular coordinates $\left(w_{1}, w_{2}\right)$. Let us change to polar:

Then

$$
\begin{aligned}
v_{1}=r \cos \theta, & v_{2}=r \sin \theta \\
w_{1}=\rho \cos \phi, & w_{2}=\rho \sin \phi
\end{aligned}
$$

$$
\xi(\rho, \phi)=\int_{\theta=0}^{2 \pi} \int_{r=0}^{\infty} e^{-r^{2}} r^{2} \log \left(r^{2}\right) \cos (2 \pi r \rho \cos (\theta-\phi)) r d r d \theta
$$

This does not depend on $\phi$, so we take $\phi=0$, getting

$$
\xi(\rho)=2 \pi \int_{r=0}^{\infty} e^{-r^{2}} r^{3} \log \left(r^{2}\right) J_{0}(2 \pi r \rho) d r
$$

in which $J_{0}$ is the Bessel function

$$
J_{0}(z)=\frac{1}{\pi} \int_{\theta=0}^{\pi} \cos (z \cos \theta) d \theta
$$

From (5) we obtain

$$
\begin{aligned}
\sum-\int= & \left(\pi \sum_{\substack{k_{1}, k_{2}=-\infty \\
\left(k_{1}, k_{2}\right) \neq(0,0)}}^{\infty} \exp \left(-\left(\pi^{2} k_{1}^{2} t^{-2}+\pi^{2} k_{2}^{2} t^{-2}\right)\right)\right) \\
& -\left(\varepsilon \sum_{\substack{k_{1}, k_{2}=-\infty \\
\left(k_{1}, k_{2}\right) \neq(\mathbf{0}, 0)}}^{\infty} \xi\left(\frac{1}{t} \sqrt{k_{1}^{2}+k_{2}^{2}}\right)\right)+O\left(\varepsilon^{2} t^{3}\right) .
\end{aligned}
$$


Now we put

$$
t=\frac{\pi}{\sqrt{\log (1 / \varepsilon)}}
$$

which makes the second term on the right-hand side much more significant than either the first term or the error term, so that

$$
\sum-\int=-(1+o(1)) \varepsilon \sum_{\substack{k_{1}, k_{2}=-\infty \\\left(k_{1}, k_{2}\right) \neq(0,0)}}^{\infty} \xi\left(\frac{\sqrt{\log (1 / \varepsilon)}}{\pi} \sqrt{k_{1}^{2}+k_{2}^{2}}\right)
$$

as $\varepsilon \rightarrow 0+$.

Consider $\xi(\rho)$ when $\rho$ is large. From [1, p. 364, eqn. 9.2.1], we have

$$
J_{0}(z)=\sqrt{\frac{2}{\pi z}} \cos \left(z-\frac{\pi}{4}\right)+O\left(z^{-3 / 2}\right)
$$

Applying the method described by Erdélyi $([5, \S 2.6])$ to the integral (6), we get $\xi(\rho)=O\left(\rho^{-5 / 2}\right)$ as $\rho \rightarrow \infty$. Also $\xi(\rho)>0$ for $\rho>0.573314$. Consequently, for all sufficiently small $\varepsilon>0$, the double sum on the right-hand side of (7) converges to a positive number. Thus $\sum-\int$ is negative for small enough $\varepsilon>0$. By (4), we have $\int / \sum>1$, and the proof is complete.

\section{REFERENCES}

1. M. Abramowitz and I. A. Stegun, eds., Handbook of Mathematical Functions (National Bureau of Standards, 1965; and Dover 1966).

2. J. W. S. Cassels, An Introduction to the Geometry of Numbers (Springer-Verlag, N.Y., 1959).

3. J. H. Conway and N. J. A. Sloane, Sphere Packings, Lattices and Groups (Springer-Verlag, N.Y., 1987).

4. N. D. Elkies, A. M. Odlyzko and J. A. Rush, On the packing densities of superballs and other bodies, Invent. Math. 105 (1991), 613-639.

5. A. Erdélyi, Asymptotic Expansions (Dover, 1956).

6. P. M. Gruber and C. G. LekKerkerker, Geometry of Numbers (Elsevier, North-Holland, Amsterdam, 1987).

7. E. Hlawka, Zur Geometrie der Zahlen, Math. Z. 49 (1943), 285-312.

8 H. Minkowski, Geometrie der Zahlen (I. Leipzig, B. G. Teubner, 1896).

9. H. MinkowsKI, Gesammelte Abhandlungen (Chelsea, N.Y. (reprint), 1969).

10. C. A. Rogers, Packing and Covering (Cambridge University Press, 1964).

11. J. A. Rush and N. J. A. SloAne, An improvement to the Minkowski-Hlawka bound for packing superballs, Mathematika 34 (1987), 8-18.

12. J. A. Rush, A lower bound on packing density, Invent. Math. 98 (1989), 499-509. 
13. J. A. Rush, A bound, and a conjecture, on the maximum lattice-packing density of a superball, Mathematika 40 (1993), 137-143.

Department of Mathematics, Box 354350

UNIVERSITY OF WASHINGTON

SEATtLe, WA 98195

USA

E-mail address: jar@math.washington.edu 